\title{
Dacomitinib improves chemosensitivity of cisplatin-resistant human ovarian cancer cells
}

\author{
LEI XU, YING XU, JIANBING ZHENG, YUN ZHAO, HONGCAI WANG and YUSHU QI
}

Obstetrics and Gynecology Department, Maternal and Child Health Hospital of Zibo City, Zibo, Shandong 255022, P.R. China

Received May 4, 2020; Accepted January 28, 2021

DOI: 10.3892/ol.2021.12830

\begin{abstract}
Drug resistance hinders effectiveness of human ovarian cancer (OC) therapies, such as cisplatin or paclitaxel therapy. Although dacomitinib, a novel anticancer agent is used against multiple types of cancers, such as non-small cell lung cancer, head and neck cancer, few studies report its effectiveness in drug-resistant human OC cells. In the present study, would healing, microplate spectrophotometer analysis, flow cytometry analysis, western blotting and Gene Expression Omnibus (GEO) analysis were used to detect the synergistic effect of dacomitinib and cisplatin in human OC SKOV-3 or OV-4 cells. Co-administration of dacomitinib and cisplatin significantly reduced viability and promoted cell apoptosis of drug resistant OC cells. In addition, dacomitinib increased Cadherin 1 (CDH1) levels and decreased P-glycoprotein (P-GP) levels in cisplatin-resistant OC cells. In addition, GEO analysis demonstrated that dacomitinib inhibited the epidermal growth factor receptor (EGFR) signaling pathway. In summary, dacomitinib improves chemosensitivity of cisplatin in human $\mathrm{OC}$ by regulating $\mathrm{CDH} 1$ and $\mathrm{P}-\mathrm{GP}$ protein levels and inhibiting the EGFR signaling pathway.
\end{abstract}

\section{Introduction}

Human ovarian cancer (OC) is one of the most lethal types of cancer in women (1). A total of 239,000 patients are diagnosed with OC and 152,000 OC-related deaths are reported worldwide every year (2). OC is a heterogeneous disease comprising a collection of neoplasms with distinct clinic morphological and molecular heterogeneity (3). Current OC therapy includes optimal primary cytoreductive surgery and

Correspondence to: Dr Lei Xu or Dr Yushu Qi, Obstetrics and Gynecology Department, Maternal and Child Health Hospital of Zibo City, 11 Xingyuan Dong Road, Zibo, Shandong 255022, P.R. China

E-mail: x11976@126.com

E-mail: qiqi940552@qq.com

Key words: drug resistance, human ovarian cancer, dacomitinib, cisplatin, cadherin 1, P-glycoprotein, epidermal growth factor receptor signaling pathway systemic chemotherapies comprising taxanes (paclitaxel) and platinum (cisplatin or carboplatin) compounds (4). Inspite significant advances in surgery and chemotherapy for OC over the last two decades, this cancer is associated with poor overall survival of patients (5). In addition, traditional therapy is characterized by severe side effects and increased drug resistance, hence novel drugs with higher efficacy should be explored (6). Furthermore, novel personalized therapies should be developed to improve efficacy in patients with OC. MicroRNAs are implicated in progression of OC (7). However, a previous study reported microRNA mediated drug resistance in OC (8). Hence, safety and specificity of microRNAs based therapeutics needs to be explored further.

Vascular endothelial growth factor, rapamycin (mTOR), and epidermal growth factor receptor (EGFR) signaling pathways have been explored extensively for development of OC therapies (9). Combination of inhibitors targeting these pathways demonstrates good synergistic effects in patients with OC (10). EGFR is an important type of receptor tyrosine kinase and the EGFR pathway contains epidermal growth factor receptor Her1 (EGFR; ErbB1), Her2 (ErbB2), Her3 (ErbB3), and Her4 (ErbB4) signaling molecules. In our previous study, we reported that increased expression of EGFR is associated with poor prognosis of patients with OC pathogenesis (11). High expression levels of ErbB3 and therapeutic regimen targeting ErbB3 were reported in OC cells (12). High expression levels of ErbB3/Neuregulin 1 in OC cells is implicated in promotion of omentum metastasis (13). In addition, miR-152 suppresses OC cell proliferation, migration and invasion, and promotes apoptosis by inhibiting ErbB3 (14). Recently, ErbB3 was reported as a potential target in OC treatment (12). A previous study has reported that 35-70\% of patients with OC with upregulated EGFR expression have a poor prognosis (9). Hence, EGFR is a potential therapeutic target for OC. First-generation EGFR tyrosine kinase inhibitors (TKIs), such as erlotinib (15) and gefitinib (16) have poor efficacy against OC. Second-generation EGFR-TKIs, such as dacomitinib (17) are more potent EGFR tyrosine kinase inhibitors compared with first-generation inhibitors. Currently, only a few functional assays and combination studies of dacomitinib with other drugs have been performed against OC $(11,18)$. Cisplatin is used for OC treatment (19). However, Nuclear factor, erythroid 2 like 2 induced cisplatin resistance in OC by promoting CD99 expression lowers effectiveness of cisplatin (20). To the best of our knowledge, to date no study has explored the activity of a combination of dacomitinib and cisplatin on OC cells. 
The aim of the present study was to explore cytotoxicity and the mechanism of action of dacomitinib against OC cells. This article provides direct evidence that dacomitinib effectively improved chemosensitivity of cisplatin-resistant human ovarian cancer cells and expands understanding of dacomitinib application. These data provide a clue of how to effectively kill resistant OC cells. However, further studies should be performed to confirm for the findings of the present study and provide information on development of effective OC therapy.

\section{Materials and methods}

Cell lines and culture conditions. SKOV-3, a human ovarian cancer cell line was obtained from ATCC. The SKOV3-DDP cell line, which was resistant to cisplatin was obtained from The Hospital Central Laboratory of Qingdao University (Qingdao, China). OV4 cells (meaning the OVCAR-4 cell line in the present study) were obtained from the Type Culture Collection of the Chinese Academy of Sciences. SKOV-3 and SKOV3-DDP cells were cultured in DMEM (Gibco; Thermo Fisher Scientific Inc.) containing 10\% (v/v) FBS (Gibco; Thermo Fisher Scientific Inc.) at $37^{\circ} \mathrm{C}$ with $5 \% \mathrm{CO}_{2}$, whereas OV4 cells were cultured in RIPM 1640 (Gibco; Thermo Fisher Scientific Inc.). $100 \mathrm{IU} / \mathrm{ml}$ penicillin and $10 \mathrm{mg} / \mathrm{ml}$ streptomycin were added for all sterile cell culturing.

Wound healing assay. SKOV-3 and OV4 cells (4x105/well) were plated into 6-well plates and incubated for $48 \mathrm{~h}$ in DMEM or RIPM 1640 containing $10 \%$ (v/v) FBS at $37^{\circ} \mathrm{C}$ with $5 \% \mathrm{CO}_{2}$. The cell layer was scratched with a pipette tip to create a fresh wound in the middle of the wells. Subsequently, FBS-free medium was used for the wound healing assay. Different concentrations $(0,0.3,1$ and $3 \mu \mathrm{M})$ of dacomitinib (cat. no. C9154; Selleck Chemicals) were added into respective wells. After culturing for 0,24 and $48 \mathrm{~h}$ at $37^{\circ} \mathrm{C}$ with $5 \% \mathrm{CO}_{2}$, photos of each well were taken to determine cell migration.

Cytotoxicity assay for cells treated with dacomitinib and cisplatin. SKOV-3 and SKOV3-DDP cells (5x10/well) were plated in 96-well plates with $100 \mu 1$ RPMI 1640. Cells were first treated with $1 \mu \mathrm{M}$ dacomitinib for $24 \mathrm{~h}$, then $0,2.5,5$, $10,20,40$, and $80 \mu \mathrm{M}$ of cisplatin (DDP) (cat. no. 15663-27-1; Selleck Chemicals) was added into the wells and cultured at $37^{\circ} \mathrm{C}$ with $5 \% \mathrm{CO}_{2}$. After $48 \mathrm{~h}, 20 \mu \mathrm{l} \mathrm{MTT}$ reagent $(5 \mathrm{mg} / \mathrm{ml}$; $\mathrm{pH}=7.4$ ) was added to the cell culture and left for $4 \mathrm{~h}$, following which $150 \mu \mathrm{l}$ DMSO was added for $10 \mathrm{~min}$ avoiding light. Optical density (OD) value of each well was determined at $490 \mathrm{~nm}$ using an universal microplate spectrophotometer.

Cell apoptosis analysis using fluorescence-activated cell sorting. SKOV3-DDP $\left(1 \times 10^{6}\right)$ cells treated with or without dacomitinib and cisplatin were cultured for $24 \mathrm{~h}$ at $37^{\circ} \mathrm{C}$ with $5 \% \mathrm{CO}_{2}$. After washing 3 times with PBS, cells were stained with a kit containing Annexin V-PE and 7AAD (cat. no. SY0479; Beijing Biolab Technology Co., Ltd.). Subsequently, cells were resuspended with $0.2 \mathrm{ml} \mathrm{PBS}$ and analyzed using the fluorescence-activated cell sorting technique using a FACSCantoII (BD Biosciences) and Flowjo v.10 (Becton, Dickinson \& Company). Both early and late stage apoptosis were assessed.
Western blotting for assessing the protein expression level of proteins associated with epithelial mesenchymal transition (EMT) or drug resistance. SKOV-3 and SKOV3-DDP cells treated with dacomitinib or cisplatin were lysed on ice for $30 \mathrm{~min}$ using mammalian cell lysis RIPA buffer (cat. no. P0013C; Beyotime Insitute of Biotechnology) containing protease and phosphatase inhibitor cocktails $(1: 1,000)$. Samples were then centrifuged at $13,000 \mathrm{x}$ g for $20 \mathrm{~min}$ at $4^{\circ} \mathrm{C}$. Protein concentration was measured by BCA kit (cat. no. P0012S; Beyotime Institute of Biotechnology). Supernatants containing $20 \mu \mathrm{g}$ proteins/lane were mixed with loading buffer for $10 \mathrm{~min}$ at $100^{\circ} \mathrm{C}$. Cell lysate proteins were then separated using 12\% SDS-PAGE gels and were transferred to PVDF membranes. Clipped PVDFs were blocked with $5 \%$ non-fat skimmed milk (dissolved in $1 \mathrm{X}$ Tris-buffered saline with $0.1 \%$ Tween-20) for $1 \mathrm{~h}$ at room temperature. PVDF membranes were then incubated with monoclonal primary antibodies against CDH1 (cat. no. Ab76055; Abcam), SLUG (snail family transcriptional repressor 2) (cat. no. Ab180714; Abcam), EGFR (cat. no. Ab52894; Abcam), P-EGFR (cat. no. Ab40815; Abcam), P-GP (cat. no. Ab103477; Abcam), and the internal reference $\beta$-actin (cat. no. 9710; Origene Technologies Inc.) overnight at $4^{\circ} \mathrm{C}$. After washing 3 times with TBST (TBS+0.05\% Tween 20), PVDF membranes were incubated with an appropriate horse radish peroxidase (HRP)-conjugated secondary antibody (cat. no. 15140-122; Gibco; Thermo Fisher Scientific Inc.), or Goat Anti-Mouse IgG H\&L (HRP) (cat. no. Ab6789; Abcam), or Goat Anti-Rabbit IgG H\&L (HRP) (cat. no. Ab6721; Abcam) respectively. DAB kit (cat. no. ZLT-9031; Origene Technologies Inc.) was used to determine protein levels. The dilutions of all the primary antibodies $(1: 1,000)$ and secondary antibodies $(1: 5,000)$ were used in the present study. Image $\mathbf{J}$ software (National Institutes of Health) was used to measure the densitometry of the grey band. CDH1 and SLUG markers were used for EMT related protein $(21,22)$. EGFR and P-GP markers were used for drug resistant protein $(23,24)$.

Determination of differentially expressed genes (DEGs) and potential proteins associated with low expression of HER2 (EGFR signaling member) in human OC using bioinformatics. GSE31432 (Illumina Human HT-12 v.3.0 expression bead chip) data (25) were retrieved from the Gene Expression Omnibus (GEO) database (https://www.ncbi.nlm.nih.gov/geo). The dataset comprised 4 types of human OC samples: negative group cells treated with trastuzumab, or pertuzumab, or both. These inhibitors reduce HER2 expression, hence have similar activity on OC cells as dacomitinib treatment. DEGs in the negative group and the drug-treatment group (trastuzumab and pertuzumab) were first determined (unpaired Student's t-test was used and genes with an adjusted P-value $<0.0004$ were defined as DEGs). Functional proteins were predicted using the String webserver (https://string-db.org/) with Gene Ontology (GO) enrichment and Kyoto Encyclopedia of Genomics and Genes (KEGG) pathway analysis.

Statistical analysis. Three or more independent experiments were performed and data were reported as means \pm SD. Statistical analysis were performed using SPSS Statistical Package v.17 (SPSS, Inc.) or GraphPad5 software (GraphPad Software Inc.). Unpaired Student's t-test and one-way ANOVA followed by the post hoc test were performed to compare means 
A
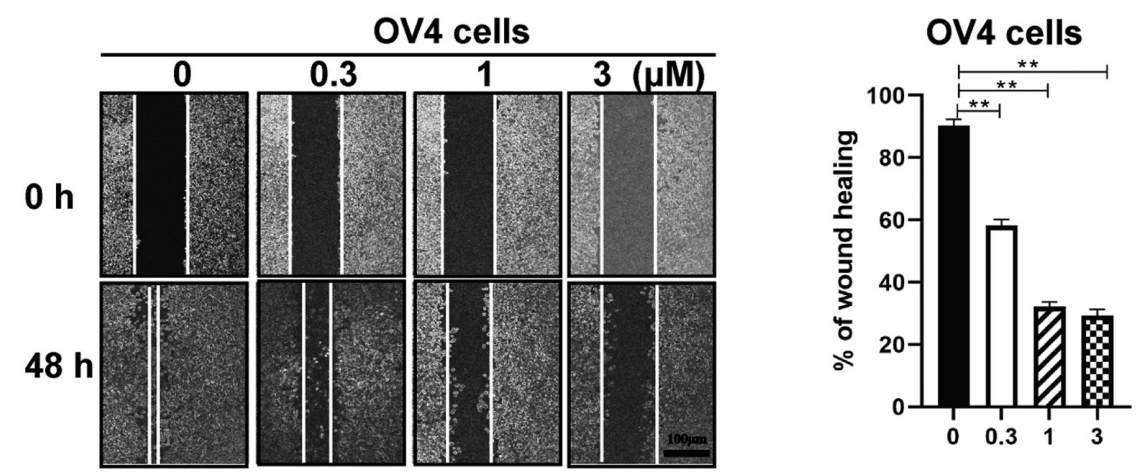

Dacomitinib concentration $(\mu \mathrm{M})$

B
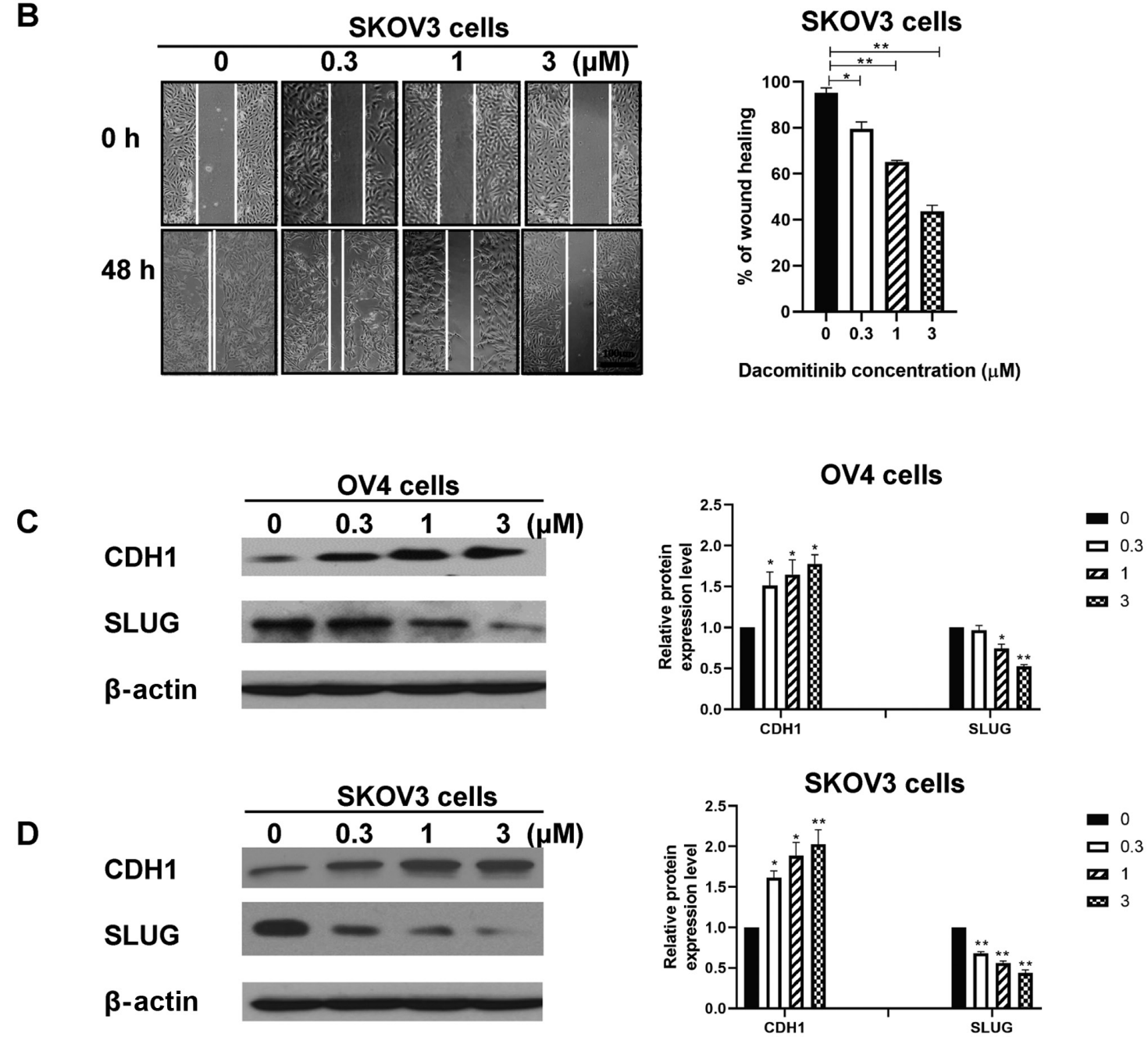

Figure 1. Migration and viability ability of human SKOV3 and OV4 cell lines following dacomitinib treatment. (A and B) Migration ability of human OV4 cells, treated with 0, 0.3, 1 and $3 \mu \mathrm{M}$ dacomitinib for $48 \mathrm{~h}$ determined by the wound healing assay. One-way ANOVA was performed. (C and D) Protein expression level of CDH1 and SLUG after dacomitinib $(0,0.3,1$ and $3 \mu \mathrm{M})$ treatment for $48 \mathrm{~h} .{ }^{*} \mathrm{P}<0.05,{ }^{* *} \mathrm{P}<0.01$. CDH1, cadherin 1; SLUG (snail family transcriptional repressor 2).

of 2 or more groups, respectively. $\mathrm{P}<0.05$ was considered to indicate a statistically significant difference.

\section{Results}

Dacomitinib inhibits migration and invasive ability of human SKOV3 and OV4 cells in a dose-dependent manner. Wound healing assay was performed to determine effect of dacomitinib treatment $(0,0.3,1,3 \mu \mathrm{M})$ on migration ability of SKOV3 and OV4 cells. The percentage of wound healing of OV4 cells treated with $3 \mu \mathrm{M}$ dacomitinib was $29.30 \%$ after $48 \mathrm{~h}$ compared with the control group which had a percentage of wound healing of $90.33 \%$ (Fig. 1A). The percentage of wound healing of SKOV 3 cells treated with $3 \mu \mathrm{M}$ dacomitinib was $43.67 \%$ after $48 \mathrm{~h}$ compared with the control group which had a percentage of wound healing of $95.33 \%$ (Fig. 1B). The 
A

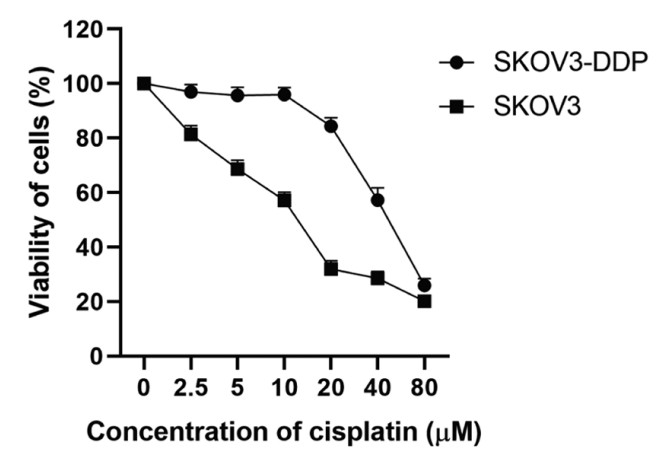

B

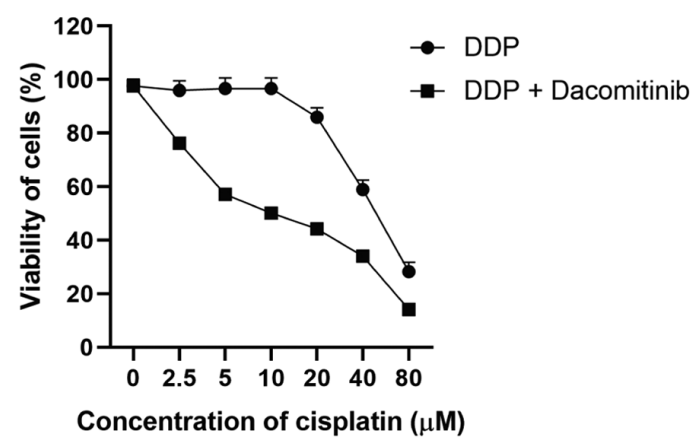

C

SKOV3-DDP

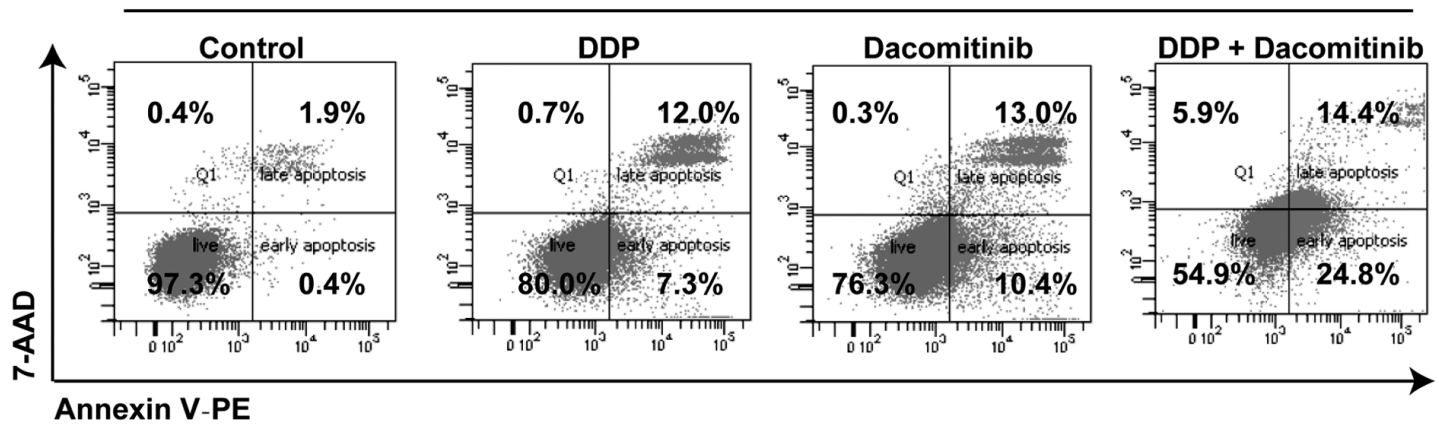

D

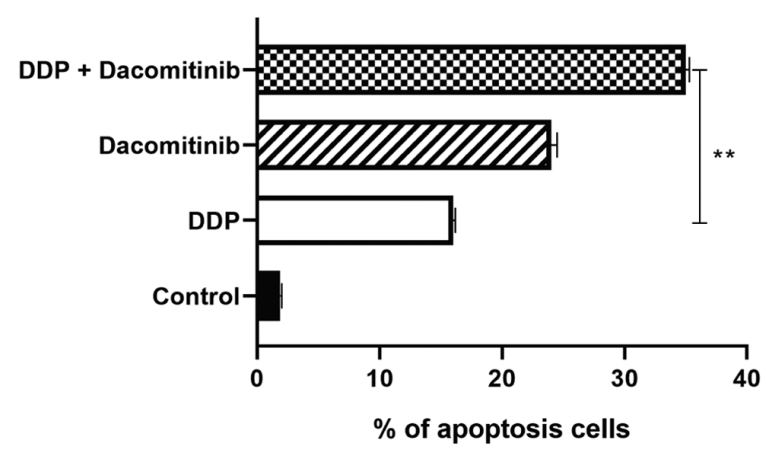

Figure 2. Viability and cell apoptosis of cisplatin-resistant ovarian cancer cells treated with dacomitinib. (A) Viability of SKOV3 and SKOV3-DDP cells after treatment with cisplatin $(0,2.5,5,10,20,40,80 \mu \mathrm{M})$ for $24 \mathrm{~h}$. (B) Viability of SKOV3-DDP cells after treatment with both dacomitinib $(1 \mu \mathrm{M})$ and cisplatin $(0,2.5,5,10,20,40,80 \mu \mathrm{M})$ for $24 \mathrm{~h}$. (C and D) Cell apoptosis of SKOV3-DDP after treatment with dacomitinib, cisplatin or both of them for $24 \mathrm{~h}$. ${ }^{* *} \mathrm{P}<0.01$. DDP, cisplatin.

aforementioned findings indicate that dacomitinib inhibits migration ability of human OC cells. High dosages of dacomitinib demonstrated higher inhibition of migration ability of OC when compared with control group (without dacomitinib) (Fig. 1A and B).

EMT related proteins, CDH1 and SLUG, were chosen to assess migration and invasive ability of human ovarian cancer cell following dacomitinib treatment. Western blotting data demonstrated increased expression of $\mathrm{CDH} 1$ and decreased expression of SLUG in SKOV3 and OV4 cells following treatment with high dacomitinib doses $(3 \mu \mathrm{M})$ and vice versa. (Fig. 1C and D). The aforementioned results indicated that dacomitinib inhibited migration and invasive ability of $\mathrm{OC}$ cells in a dose-dependent manner.

A combination of dacomitinib and cisplatin treatment inhibits viability and promotes apoptosis of human OC cells. To explore the synergistic effects of dacomitinib with other drugs, cisplatin was chosen and tested on SKOV3 and SKOV3-DDP cells. Prior to the assay, cisplatin resistant cells were developed and treated with dacomitinib. Cells were treated with cisplatin $(0,2.5,5,10,20,40$ and $80 \mu \mathrm{M})$ for $24 \mathrm{~h}$. Cells demonstrated decreased viability following treatment with a higher dosage of cisplatin $(80 \mu \mathrm{M})$ (Fig. 2A). Cisplatin had an $\mathrm{IC}_{50}$ of $12.27 \mu \mathrm{M}$ against SKOV3 cells, and $\mathrm{IC}_{50}$ of $64.34 \mu \mathrm{M}$ against SKOV3-DDP cells (drug resistance index=5.24) (data not shown).

SKOV3-DDP cells were then treated with or without dacomitinib $(1 \mu \mathrm{M})$ plus $0,2.5,5,10,20,40,80 \mu \mathrm{M}$ cisplatin for $24 \mathrm{~h}$. Cells demonstrated decreased viability following treatment with a higher dosage of cisplatin ( $80 \mu \mathrm{M})$ (Fig. 2B). An $\mathrm{IC}_{50}$ of 11.30 was observed against SKOV3-DDP cells (a 5.69 times decrease when compared with $\mathrm{IC}_{50}$ of $64.34 \mu \mathrm{M}$ against SKOV3-DDP cells) (data not shown). These findings 
A

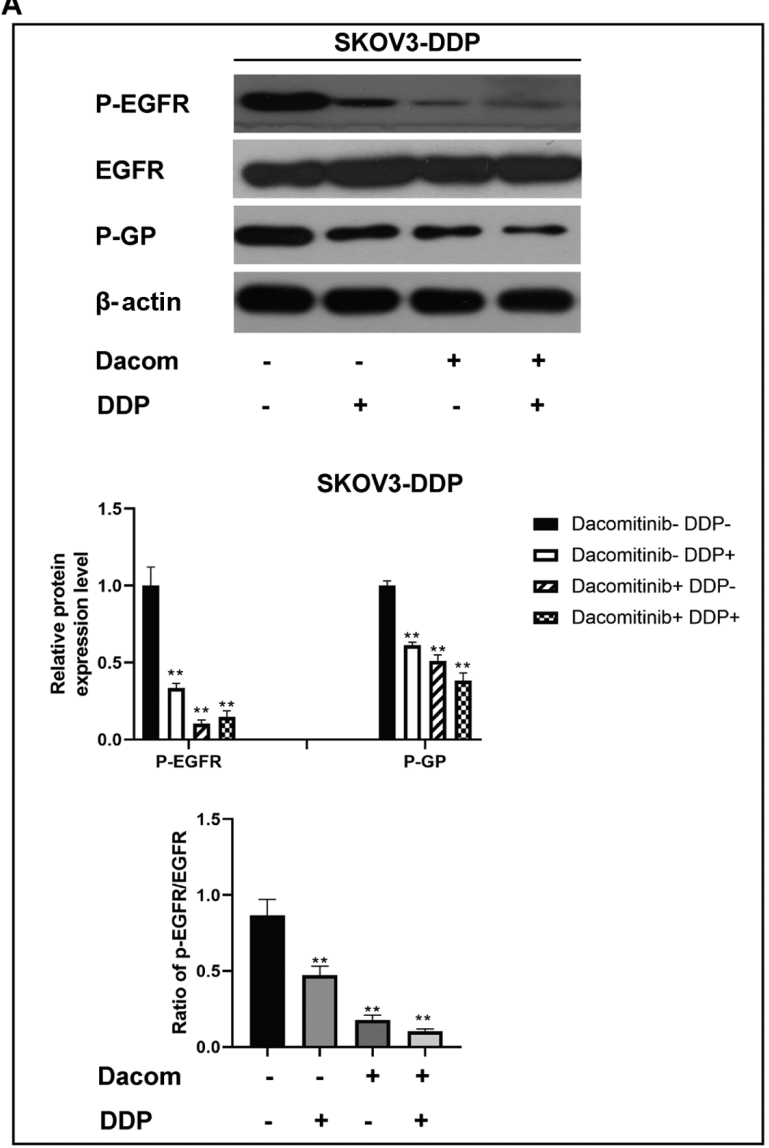

B

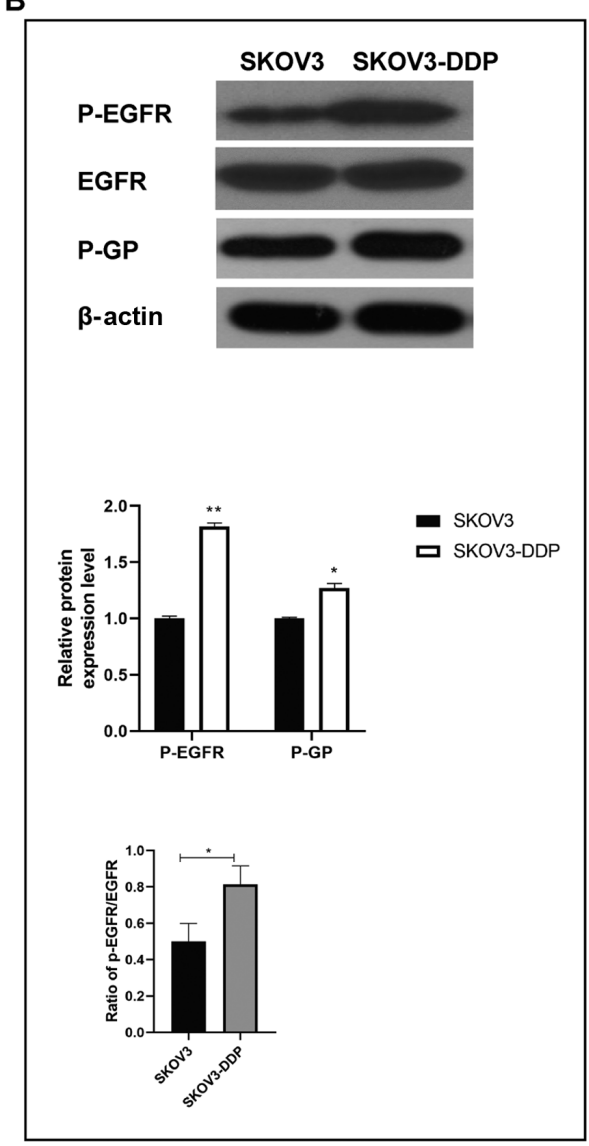

Figure 3. Protein expression level of p-EGFR and P-GP following treatment using dacomitinib in cisplatin-resistant OC cells. (A) Protein expression level of p-EGFR and P-GP in OC cells tested by western blotting following treatment with dacomitinib, cisplatin, or both of them for $24 \mathrm{~h}$. (B) Protein expression level of p-EGFR and P-GP in SKOV3 and SKOV3-DDP cells. ${ }^{*} \mathrm{P}<0.05,{ }^{* *} \mathrm{P}<0.01$. DDP, cisplatin; EGFR, epidermal growth factor receptor; p-GP, p-glycoprotein; $\mathrm{P}$, phosphorylated; OC, ovarian cancer.

implied that dacomitinib improved the antiproliferative effect of cisplatin-resistance in SKOV3-DDP cells (Fig. 2B). Apoptosis of SKOV3-DDP cells treated with dacomitinib, cisplatin, or both was tested using FACS based on Annexin V and 7AAD staining. Apoptotic assays demonstrated a higher apoptosis rate of cisplatin group (19.3\%), dacomitinib group (23.4\%), and dacomitinib plus cisplatin group $(39.2 \%)$ compared with the apoptosis rate of the control group (2.3\%). The dacomitinib plus cisplatin group demonstrated a higher percentage of apoptosis compared with the cisplatin group (DDP) (Fig. 2C and D). The aforementioned finding demonstrated that dacomitinib promotes apoptosis of cisplatin-resistant OC cells.

Dacomitinib decreases protein expression of $P$-EGFR and $P-G P$ in cisplatin-resistant $O C$ cells. P-EGFR and P-GP protein levels are associated with EGFR signaling $(26,27)$. Protein levels of p-EGFR and P-GP in SKOV3-DDP cells treated with dacomitinib and cisplatin were determined (Fig. 3A and B). Expression levels of P-EGFR and P-GP in cisplatin, dacomitinib, and dacomitinib plus cisplatin groups were significantly lower compared with that in the control group (Fig. 3A). Western blotting analysis demonstrated higher expression levels of p-EGFR and P-GP in SKOV3-DDP cells (resistant cells) compared with the levels in SKOV3 cells (Fig. 3B). These findings collectively implied that dacomitinib, an EGFR inhibitor, regulates p-EGFR and P-GP directly or indirectly to modulate growth of cisplatin-resistant OC cells.

Potential candidate genes $C D H 1$ and MMP7 are identified in decreased EGFR signaling pathway of $O C$. To further explore the effect of EGFR inhibitors in regulation of signaling pathways in human ovarian cancer cells, SKOV3 samples treated with EGFR inhibitors, such as trastuzumab and pertuzumab were retrieved from the GEO database (GSE31432). Dacomitinib, trastuzumab, and pertuzumab are EGFR and HER2 inhibitors (28-30). EGFR inhibitor-treated samples were used to determine potential genes regulated by dacomitinib. A total of 6 samples without any treatment were chosen for group A, and 5 samples treated with EGFR inhibitor trastuzumab plus pertuzumab were chosen for group B. GEO analysis demonstrated that these samples had good quality of total gene expression (data not shown). The top 100 genes were identified based on P-value (Table SI).

GO enrichment and KEGG pathway analysis were performed on these candidate genes using the String webserver. A total of 66 proteins demonstrated highly related interaction (PPI enrichment $\mathrm{P}$-value $=4.77 \times 10^{-7}$ ) from the top 100 genes (Fig. 4A). Biological process (GO) category demonstrated that only 15 proteins were enriched in regulation of cell adhesion, whereas 8 proteins were enriched in negative regulation of cell adhesion (Fig. 4B). Cellular component (GO) demonstrated 
A

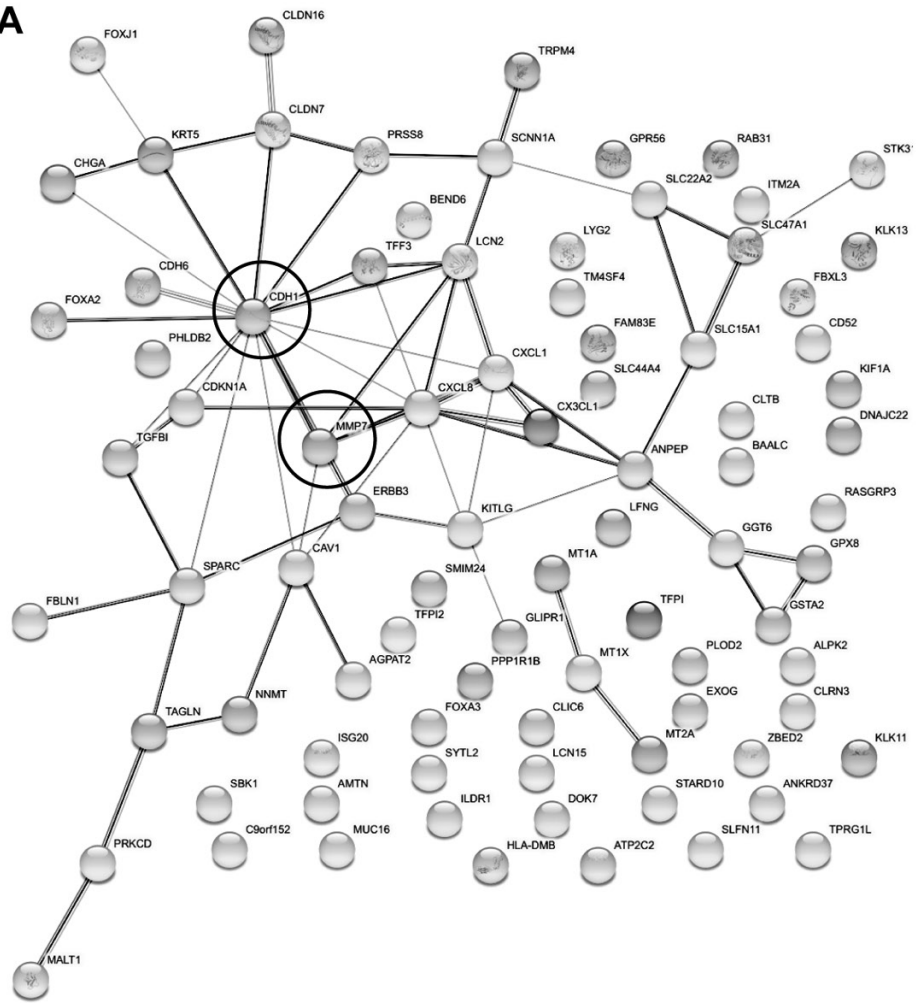

B

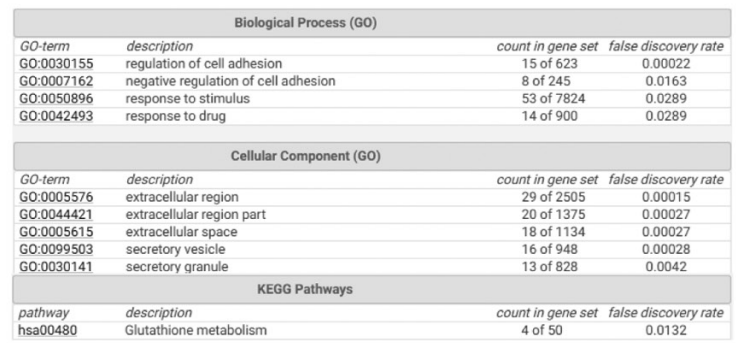

C

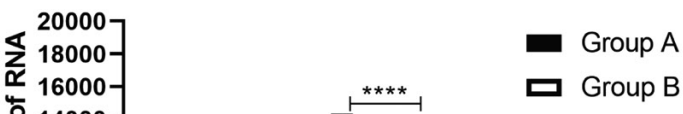

Figure 4. Potential candidate genes predicted in decreased EGFR signaling pathway of human OC cells based on GEO database and string tool analysis. (A) Protein-protein interaction networks using string tool (https://string-db.org/) in decreased EGFR signaling pathway of human OC. (B) GO enrichment and KEGG pathway analysis were performed for specific signaling pathway based on top 100 genes between group A (samples without any treatment) and B (samples treated with EGFR inhibitor trastuzumab plus pertuzumab). (C) Expression level of CDH1 and MMP7 after inhibition of EGFR signaling pathway in human ovarian cancer $(\mathrm{n}=3) .{ }^{* * * * *} \mathrm{P}<0.0001$. Student's t-test was performed. GEO, Gene Expression Omnibus; KEGG, Kyoto Encyclopedia of Genomics and Genes; CDH1, cadherin 1; MMP7, matrix metalloproteinase 7; OC, ovarian cancer; EGFR, epidermal growth factor receptor.

extracellular region (49 proteins), extracellular space (18 proteins), and secretory vesicle/granule (29 proteins) were highly enriched (Fig. 4B). KEGG pathway analysis showed 4 proteins enriched in glutathione metabolism signaling (Fig. 4B).

There were several key nodes, such as CDH1 (E-cadherin) and matrix metalloproteinase (MMP7) (Fig. 4A). Expression of CDH1 and MMP7 was detected in all samples in the GSE31432 dataset. Following EGFR inhibition, samples of human OC demonstrated increased expression level of E-cadherin and decreased expression level of MMP7 (Fig. 4C). These findings were consistent with EGFR inhibition by dacomitinib through reducing migration and invasive ability of $\mathrm{OC}$ by modulation of CDH1.

\section{Discussion}

OC drugs are characterized by low efficacy (31). Findings from our previous study demonstrated that dacomitinib reduces migration of OC cells (11). In addition, dacomotinib treatment demonstrated a significant reduction in migration of cisplatin resistance OC cells in the present study. Further transwell assays should be performed to confirm the findings of the wound healing assay. The findings of the current study demonstrated that dacomitinib decreased expression of EGFR and P-GP in OC cisplatin-resistant cells. In addition, in the present study important signaling pathways in $\mathrm{OC}$ for the samples with low expression of EGFR were predicted using
GEO analysis and String webserver. However, the role of these candidate genes in OC should be confirmed using functional assays after dacomitinib treatment in future studies.

Dacomitinib is a small-molecule inhibitor of EGFR (HER1), HER2, and HER4 (32). Dacomitinib has high cytotoxicity against multiple types of cancer cells resistant to drugs, such as EGFR inhibitor gefitinib and erlotinib $(33,34)$. Dacomitinib increases CDH1 and decreases SLUG expression levels. CDH1 and SLUG proteins are important EMT related proteins $(21,22)$. EMT related proteins serve an important role in migration of different cancer cells including OC cells (35). During EMT, epithelial-type cancer cells undergo molecular (epigenetic changes), morphological (biomechanical forces), and functional changes (invasive ability) (36). High expression levels of CDH1 is a nonmalignant tumor marker, whereas SLUG binds to the promoter of CDH1 and inhibits expression of CDH1 (37). The aforementioned studies provide evidence that dacomitinib inhibits growth of OC cells through modulation of CDH1 and SLUG expression levels.

In the present study, to determine the effectiveness of dacomitinib in drug-resistant OC cells, cells were first exposed to different concentrations of cisplatin. Cisplatin-resistant cells were then treated with dacomitinib. In the present study, dacomitinib demonstrated a significant apoptotic effect in cells exposed to high cisplatin concentrations compared with treatment of OC cells with cisplatin alone. This implied that a combination of dacomitinib and cisplatin has a synergistic effect on patients with OC. The mechanism of action of 
dacomitinib in killing drug-resistant OC cells is currently not clear (11).

P-GP (ABCB1) is a $170 \mathrm{kDa}$ transmembrane protein, expressed from the MDRl locus (38), and is associated with multiple drug resistance (39-41). A previous meta-analysis demonstrated that high expression of EGFR is associated with worse survival rates of patients with $\mathrm{OC}$, and high expression of P-GP is related with cisplatin resistance (42). Hence, in the present study EGFR and P-GP were selected to explore the function of dacomitinib in drug resistant cells. In the present study, high expression of EGFR and P-GP were observed in SKOV3-DDP, but not in SKOV3 cells. The findings of the present study implied that EGFR and P-GP may participate in progression of resistance. A recent study reported inhibition of EGFR reverses cisplatin resistance in OC (43). Cisplatin exerts its activity by targeting protein kinase $\mathrm{G}$ (PKG) (44). Overexpression of PKG2 may inhibit expression and phosphorylation of EGFR in OC (45). Cisplatin may regulate PKG2 to further inhibit EGFR in OC, however the exact mechanisms of this need to be explored. In the present study, dacomitinib treatment reduced expression of EGFR and P-GP in SKOV3-DDP cells. Hence, dacomitinib may improve chemosensitivity of cisplatin in OC cells by regulating expression of EGFR and P-GP.

Dacomitinib treated OC samples were available in the GEO database (25). In the present study, western blotting demonstrated that dacomitinib significantly reduced expression of EGFR. In the present study, samples treated with other inhibitors had a similar RNA profile as cells treated with dacomitinib. The findings of the present study revealed important signaling pathways in OC progression, such as regulation of cell adhesion, extracellular region part, vesicle, membrane-bounded vesicle, extracellular space and glutathione metabolism signaling pathways. Inhibition of EGFR expression inhibits cell adhesion signaling pathway (46). Dacomitinib may be a potential therapy for patients with OC.

The present study had several limitations. Firstly, no transwell invasion assays were used and should be performed by future studies. Secondly, no propidium iodide was used to evaluate apoptosis of cell treated with dacomitinib and cisplatin. No in vivo assays were performed in the present study. Future studies should perform these to verify the in vitro findings of the present study.

In conclusion, the present study demonstrated that dacomitinib inhibits human OC cell viability through modulation of the protein expression of $\mathrm{CDH} 1$ and P-GP. In addition, it decreases activity of the EGFR signaling pathway improving chemosensitivity of cisplatin-resistant OC cells. Further studies should be performed to explore the specific mechanism of dacomitinib effect on OC development.

\section{Acknowledgements}

Not applicable.

\section{Funding}

This study was partly supported by the Development Fund of Zibo Maternal and Child Health Hospital and the Key Research and Development Program of Zibo City (2019gy010009).

\section{Availability of data and materials}

The datasets used and/or analyzed during the current study are available from the corresponding author on reasonable request.

\section{Authors' contributions}

LX and YQ conceived, designed, performed all experiments and wrote the manuscript. LX and YQ confirm the authenticity of all the raw data. YX and YZ were responsible for the collection and follow-up of clinical cases. JZ and HW ere responsible for data statistics. All authors have read and approved the manuscript.

\section{Ethics approval and consent to participate}

Not applicable.

\section{Patient consent for publication}

Not applicable.

\section{Competing interests}

The authors declare that they have no competing interests.

\section{References}

1. Siegel RL, Miller KD, Fuchs HE and Jemal A: Cancer statistics, 2021. CA Cancer J Clin 71: 7-33, 2021.

2. Reid BM, Permuth JB and Sellers TA: Epidemiology of ovarian cancer: A review. Cancer Biol Med 14: 9-32, 2017.

3. Kossaï M, Leary A, Scoazec JY and Genestie C: Ovarian cancer: A heterogeneous disease. Pathobiology 85: 41-49, 2018.

4. Angioli R, Palaia I, Zullo MA, Muzii L, Manci N, Calcagno M and Panici PB: Diagnostic open laparoscopy in the management of advanced ovarian cancer. Gynecol Oncol 100: 455-461, 2006.

5. Ray-Coquard I, Mirza MR, Pignata S, Walther A, Romero I and du Bois A: Therapeutic options following second-line platinum-based chemotherapy in patients with recurrent ovarian cancer: Comparison of active surveillance and maintenance treatment. Cancer Treat Rev 90: 102107, 2020.

6. Kuroki L and Guntupalli SR: Treatment of epithelial ovarian cancer. BMJ 371: m3773, 2020

7. Deb B, Uddin A and Chakraborty S: miRNAs and ovarian cancer: An overview. J Cell Physiol 233: 3846-3854, 2018.

8. Mihanfar A, Fattahi A and Nejabati HR: MicroRNA-mediated drug resistance in ovarian cancer. J Cell Physiol 234: 3180-3191, 2019.

9. Dinh P, Harnett P, Piccart-Gebhart MJ and Awada A: New therapies for ovarian cancer: Cytotoxics and molecularly targeted agents. Crit Rev Oncol Hematol 67: 103-112, 2008.

10. Smolle E, Taucher V, Pichler M, Petru E, Lax S and Haybaeck J: Targeting signaling pathways in epithelial ovarian cancer. Int $\mathrm{J}$ Mol Sci 14: 9536-9555, 2013.

11. Xu L, Wu H, Jiang C, Wang H, Gao B, Yan S, Qi Y and Zhou S: Dacomitinib, a new pan-EGFR inhibitor, is effective in killing ovarian cancer cells. Discov Med 22: 297-309, 2016.

12. Camblin AJ, Tan G, Curley MD, Yannatos I, Iadevaia S, Rimkunas V, Mino-Kenudson M, Bloom T, Schoeberl B, Drummond DC, et al: Dual targeting of IGF-1R and ErbB3 as a potential therapeutic regimen for ovarian cancer. Sci Rep 9: $16832,2019$.

13. PradeepS,Kim SW,WuSY,NishimuraM,Chaluvally-RaghavanP Miyake T, Pecot CV, Kim SJ, Choi HJ, Bischoff FZ, et al: Hematogenous metastasis of ovarian cancer: Rethinking mode of spread. Cancer Cell 26: 77-91, 2014.

14. Li LW, Xiao HQ, Ma R, Yang M, Li W and Lou G: miR-152 is involved in the proliferation and metastasis of ovarian cancer through repression of ERBB3. Int J Mol Med 41: 1529-1535, 2018. 
15. Gordon AN, Finkler N, Edwards RP, Garcia AA, Crozier M, Irwin DH and Barrett E: Efficacy and safety of erlotinib $\mathrm{HCl}$, an epidermal growth factor receptor (HER1/EGFR) tyrosine kinase inhibitor, in patients with advanced ovarian carcinoma: Results from a phase II multicenter study. Int J Gynecol Cancer 15: 785-792, 2005.

16. Schilder RJ, Sill MW, Chen X, Darcy KM, Decesare SL, Lewandowski G, Lee RB, Arciero CA, Wu H and Godwin AK: Phase II study of gefitinib in patients with relapsed or persistent ovarian or primary peritoneal carcinoma and evaluation of epidermal growth factor receptor mutations and immunohistochemical expression: A gynecologic oncology group study. Clin Cancer Res 11: 5539-5548, 2005.

17. Liao BC, Lin CC and Yang JC: Second and third-generation epidermal growth factor receptor tyrosine kinase inhibitors in advanced nonsmall cell lung cancer. Curr Opin Oncol 27: 94-101, 2015.

18. Momeny M, Zarrinrad G, Moghaddaskho F, Poursheikhani A, Sankanian G, Zaghal A, Mirshahvaladi S, Esmaeili F, Eyvani H, Barghi F, et al: Dacomitinib, a pan-inhibitor of ErbB receptors, suppresses growth and invasive capacity of chemoresistant ovarian carcinoma cells. Sci Rep 7: 4204, 2017.

19. Dasari S and Tchounwou PB: Cisplatin in cancer therapy: Molecular mechanisms of action. Eur J Pharmacol 740: 364-378, 2014.

20. Wu J, Zhang L, Li H, Wu S and Liu Z: Nrf2 induced cisplatin resistance in ovarian cancer by promoting CD99 expression. Biochem Biophys Res Commun 518: 698-705, 2019.

21. Li R, Ong SL, Tran LM, Jing Z, Liu B, Park SJ, Huang ZL, Walser TC, Heinrich EL, Lee G, et al: Chronic IL-1beta-induced inflammation regulates epithelial-to-mesenchymal transition memory phenotypes via epigenetic modifications in non-small cell lung cancer. Sci Rep 10: 377, 2020.

22. Miao Y, Liu G and Liu L: Histone methyltransferase SUV39H2 regulates LSD1-dependent CDH1 expression and promotes epithelial mesenchymal transition of osteosarcoma. Cancer Cell Int 21: 2, 2021

23. Zhao L, Fan T, Shi Z, Ding C, Zhang C, Yuan Z, Sun Q, Tan C, Chu B and Jiang Y: Design, synthesis and evaluation of novel ErbB/HDAC multitargeted inhibitors with selectivity in EGFR $^{\mathrm{T} 790 \mathrm{M}}$ mutant cell lines. Eur J Med Chem 213: 113173, 2021.

24. Hyokai S, Tanaka H, Aihara N and Kamiie J: Expression of P-glycoprotein and breast cancer resistance protein in three cases of canine lymphoma showing drug resistance. J Vet Med Sci, Jan 29, 2021 (Epub ahead of print).

25. Sims AH, Zweemer AJ, Nagumo Y, Faratian D, Muir M, Dodds M, Um I, Kay C, Hasmann M, Harrison DJ and Langdon SP: Defining the molecular response to trastuzumab, pertuzumab and combination therapy in ovarian cancer. Br J Cancer 106: 1779-1789, 2012

26. Toolabi M, Moghimi S, Bakhshaiesh TO, Salarinejad S, Aghcheli A, Hasanvand Z, Nazeri E, Khalaj A, Esmaeili R and Foroumadi A: 6-Cinnamoyl-4-arylaminothienopyrimidines as highly potent cytotoxic agents: Design, synthesis and structure-activity relationship studies. Eur J Med Chem 185: 111786, 2020.

27. Lu ZN, Shi ZY, Dang YF, Cheng YN, Guan YH, Hao ZJ, Tian B, He HW and Guo XL: Pantoprazole pretreatment elevates sensitivity to vincristine in drug-resistant oral epidermoid carcinoma in vitro and in vivo. Biomed Pharmacother 120: 109478, 2019.

28. Hao Sun and Wu YL: Dacomitinib in non-small-cell lung cancer: A comprehensive review for clinical application. Future Oncol 15: 2769-2777, 2019.

29. Hurvitz SA, Caswell-Jin JL, McNamara KL, Zoeller JJ, Bean GR, Dichmann R, Perez A, Patel R, Zehngebot L, Allen H, et al: Pathologic and molecular responses to neoadjuvant trastuzumab and/or lapatinib from a phase II randomized trial in HER2-positive breast cancer (TRIO-US B07). Nat Commun 11: 5824,2020

30. Yamashita T, Masuda N, Saji S, Araki K, Ito Y, Takano T, Takahashi M, Tsurutani J, Koizumi K, Kitada M, et al: Trastuzumab, pertuzumab, and eribulin mesylate versus trastuzumab, pertuzumab, and a taxane as a first-line or second-line treatment for HER2-positive, locally advanced or metastatic breast cancer: Study protocol for a randomized controlled, non-inferiority, phase III trial in Japan (JBCRG-M06/EMERALD). Trials 21: 391, 2020.
31. Huang $X$ and Tang J: Human la protein: An RNA-binding protein involved in ovarian cancer development and multidrug resistance. Onco Targets Ther 13: 10721-10727, 2020.

32. Passaro A, Mok T, Peters S, Popat S, Ahn MJ and de Marinis F: Recent advances on the role of EGFR tyrosine kinase inhibitors in the management of NSCLC with uncommon, non exon 20 insertions, EGFR mutations. J Thorac Oncol S1556-0864: 31102-31103, 2020 (Epub ahead of print).

33. Engelman JA, Zejnullahu K, Gale CM, Lifshits E, Gonzales AJ, Shimamura T,Zhao F, Vincent PW, Naumov GN, Bradner JE, et al: PF00299804, an irreversible pan-ERBB inhibitor, is effective in lung cancer models with EGFR and ERBB2 mutations that are resistant to gefitinib. Cancer Res 67: 11924-11932, 2007.

34. Gonzales AJ, Hook KE, Althaus IW, Ellis PA, Trachet E, Delaney AM, Harvey PJ, Ellis TA, Amato DM, Nelson JM, et al: Antitumor activity and pharmacokinetic properties of PF-00299804, a second-generation irreversible pan-erbB receptor tyrosine kinase inhibitor. Mol Cancer Ther 7: $1880-1889,2008$

35. Kudo-Saito C, Ozaki Y, Imazeki H, Hayashi H, Masuda J, Ozawa $\mathrm{H}$ and Ogiwara Y: Targeting oncoimmune drivers of cancer metastasis. Cancers (Basel) 13: 554, 2021.

36. Klymenko Y, Kim O and Stack MS: Complex determinants of epithelial: Mesenchymal phenotypic plasticity in ovarian cancer. Cancers (Basel) 9: 104, 2017.

37. Ahmed AR and Muhammad EM: E-cadherin and CD10 expression in atypical hyperplastic and malignant endometrial lesions. J Egypt Natl Canc Inst 26: 211-217, 2014.

38. Gottesman MM and Pastan I: Biochemistry of multidrug resistance mediated by the multidrug transporter. Annu Rev Biochem 62: 385-427, 1993.

39. Goldstein LJ, Galski H, Fojo A, Willingham M, Lai SL, Gazdar A, Pirker R, Green A, Crist W, Brodeur GM, et al: Expression of a multidrug resistance gene in human cancers. J Natl Cancer Inst 81: 116-124, 1989.

40. Muthiah D, Henshaw GK, DeBono AJ, Capuano B, Scammells PJ and Callaghan R: Overcoming P-glycoprotein-mediated drug resistance with noscapine derivatives. Drug Metab Dispos 47: 164-172, 2019.

41. Chen Q, Liu X, Luo Z, Wang S, Lin J, Xie Z, Li M, Li C, Cao H, Huang Q, et al: Chloride channel-3 mediates multidrug resistance of cancer by upregulating P-glycoprotein expression. J Cell Physiol 234: 6611-6623, 2019.

42. Xu L, Cai J, Yang Q, Ding H, Wu L, Li T and Wang Z: Prognostic significance of several biomarkers in epithelial ovarian cancer: A meta-analysis of published studies. J Cancer Res Clin Oncol 139: 1257-1277, 2013.

43. Poursheikhani A, Yousefi H, Tavakoli-Bazzaz J and Seyed HG: EGFR blockade reverses cisplatin resistance in human epithelial ovarian cancer cells. Iran Biomed J 24: 370-378, 2020.

44. Perrotta C, Cervia D, Di Renzo I, Moscheni C, Bassi MT, Campana L, Martelli C, Catalani E, Giovarelli M, Zecchini S, et al: Nitric oxide generated by tumor-associated macrophages is responsible for cancer resistance to cisplatin and correlated with syntaxin 4 and acid sphingomyelinase inhibition. Front Immunol 9: 1186, 2018.

45. Xu H, Zhang Z, Li P, Lu X, Chen B and Lan T: Expression of PKG2 in ovarian cancer and its effect on epidermal growth factor receptor. J BUON 25: 729-735, 2020.

46. Pang J, Jiang P, Wang Y, Jiang L, Qian H, Tao Y, Shi R, Gao J, Chen $\mathrm{Y}$ and $\mathrm{Wu} \mathrm{Y}$ : Cross-linked hyaluronan gel inhibits the growth and metastasis of ovarian carcinoma. J Ovarian Res 11: 22,2018

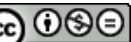

This work is licensed under a Creative Commons Attribution-NonCommercial-NoDerivatives 4.0 International (CC BY-NC-ND 4.0) License. 